\title{
Study of Nuclear Structure of Even-Even Dy Isotopes
}

\author{
Aziz Mohammad Ali ${ }^{*}$, Yasir Yahya Kassim², Mutasim M. Yosuf ${ }^{3}$ \\ ${ }^{1 *}$ Department of Educational and Psychological Sciences, College of Education for Human Sciences, \\ University of Mosul, Mosul, Iraq \\ ${ }^{2,3}$ Department of Physics, College of Education for Pure Sciences, University of Mosul, Mosul, Iraq \\ E-Mail: ${ }^{1 * d r . a z i z @ u o m o s u l . e d u . i q, ~}{ }^{2}$ yaser-phsics@uomosul.edu.iq, ${ }^{3}$ mutasim.m.u@ uomosul.edu.iq
}

(Received February 02, 2021; Accepted March 30, 2021; Available online September 01, 2021)

DOI: 10.33899/edusj.2021.129475.1140, (C) 2021, College of Education for Pure Science, University of Mosul.

This is an open access article under the CC

\begin{abstract}
In this paper, the energy of the first exited $2_{1}^{+}$and the ratios of $\frac{E\left(4_{1}^{+}\right)}{E\left(2_{1}^{+}\right)}, \frac{E\left(8_{1}^{+}\right)}{E\left(2_{1}^{+}\right)}, \frac{E\left(6_{1}^{+}\right)}{E\left(2_{1}^{+}\right)}$ for ${ }^{152-156} \mathrm{DY}$ even-even isotopes are compared with the standard values for the three limits, the vibration $\mathrm{U}(5)$, gamma-soft $\mathrm{O}(6)$ and the rotational $\mathrm{SU}(3)$. The back-bending curve and the relation between E $\gamma / \mathrm{I}$ as a function of I ( E-GOS), the ratio $E\left(I_{1}^{+}\right) / E\left(2_{1}^{+}\right)$and the ratio $\mathrm{r}(\mathrm{I}+2 / \mathrm{I})$ as a function of spin (I) are drawn for these isotopes to have more information about their properties. Calculation of the energy of different states along the yrast band has been done using the standard relations for each limit, $\mathrm{U}(5), \mathrm{O}(6)$ and $\mathrm{SU}(3)$ and a comparison with the experimental data show that they the isotopes 152 ${ }^{154} \mathrm{DY}$ have vibration $\mathrm{U}(5)$ properties and ${ }^{156} \mathrm{DY}$ has gamma-soft $\mathrm{O}(6)$ properties. The interacting boson model IBM-1,BM and IVBM has been used to calculate the energy of different states along the yrast region applying a suitable limit for each isotopes. A program with MATLAB 10 has been built for this purpose. Agood agreement with the experimental data was obtained.
\end{abstract}

keyword: Dy isotopes, IBM-1, Nuclear structure, even-even nuclei, E-GOS;back-bending, ratio $\mathrm{r}$ $(\mathrm{I}+2 / \mathrm{I})$, ratio $\mathrm{E}_{\mathrm{I}} / \mathrm{E}_{2}$

$$
\begin{aligned}
& \text { دراسة التركيب النووية لنظائر Dy الزوجية_الزوجية }
\end{aligned}
$$

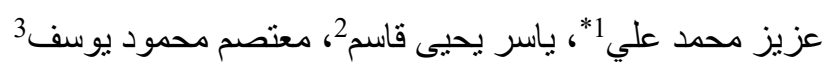

$$
\begin{aligned}
& \text { 1" قسم علوم التربوية والنفسية_كلية التربية للعلوم الإنسانيةـ جامعة الموصل_الموصل-العرق } \\
& \text { 2،3 قسم الفيزياء-كلية التربية للعلوم الصرفة-جامعة الموصل_الموصل_العراق }
\end{aligned}
$$


في هذه الدراسة، تم مقارنة مواقع حالات طاقة الاثارة لطاقة المستوي الأول E(2) E(2) والنسب بين الطاقات المثارة الى طاقة المستوي الأول في لن الناه

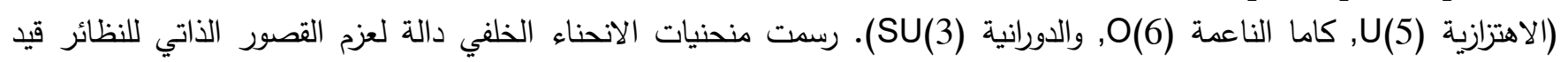

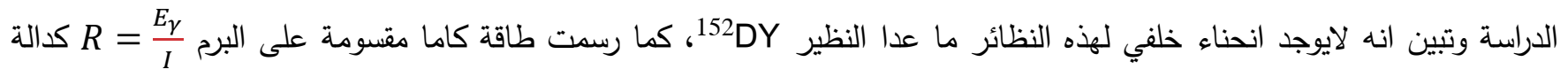

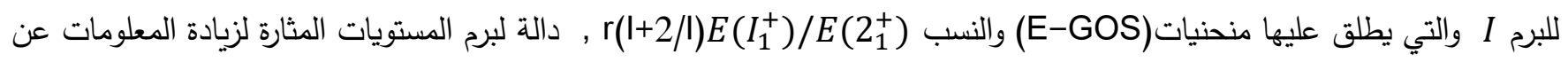

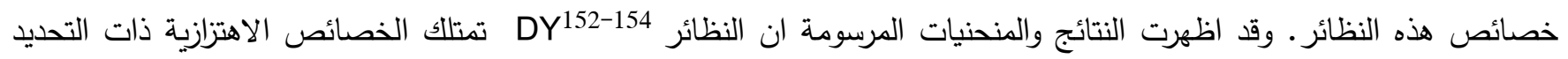

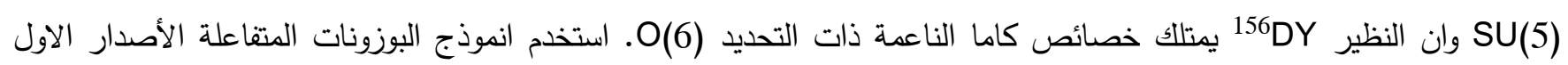

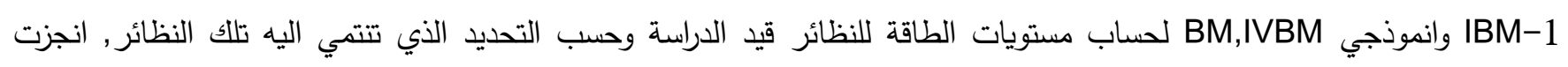
جميع الحسابات من خلال بناء برنامج بلغة MATLAB-10 وتم مقارنة النتائج المحسوبة مع القيم العملية وكانت جيدة. الكلمات المفتاحية: نظائر Dy, أنموذج البوزونات المتفاعلة، التركيب النووي, النوى الزوجية-الزوجية، منحنيات GOS، الانحناء

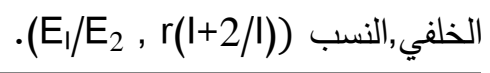

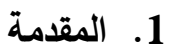

تعد النواة منظومة كمية تحتوي على مجموعة من النكليونات تثكل قلبا هامدا باحد الاعداد السحرية للنكليونات خارج القلب الهامد تتفاعل

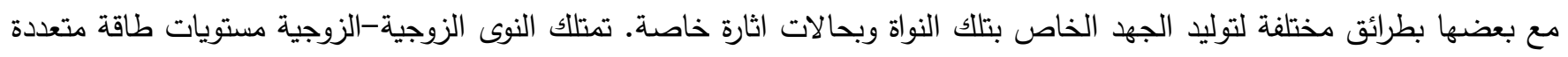

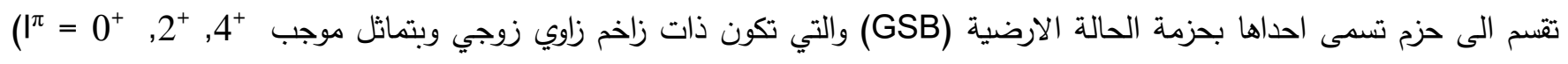

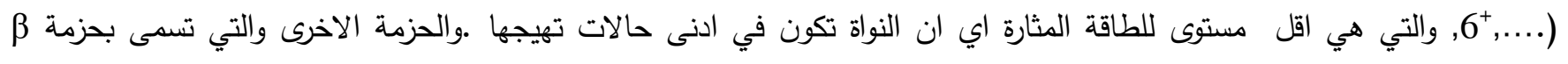

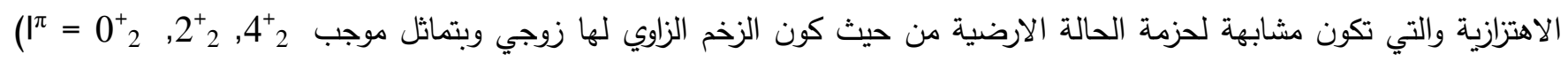

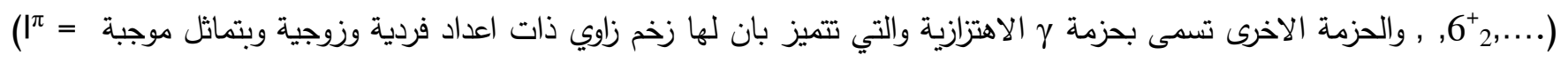

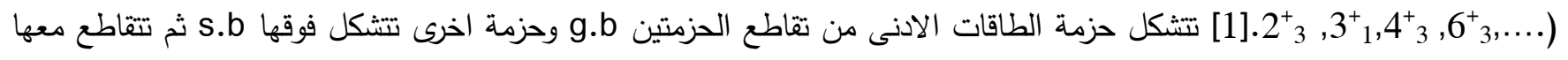

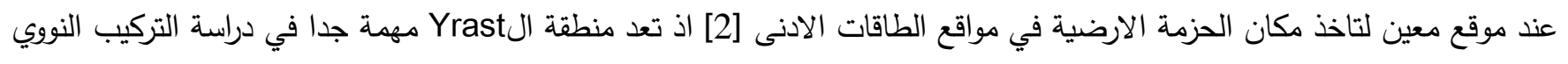

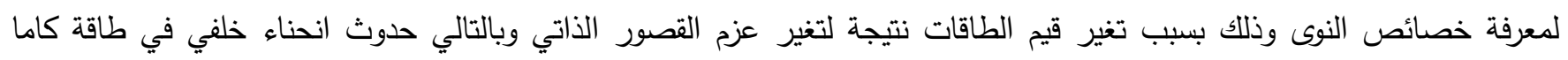

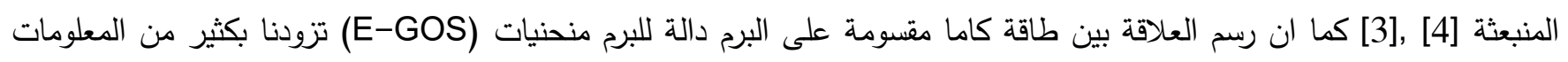

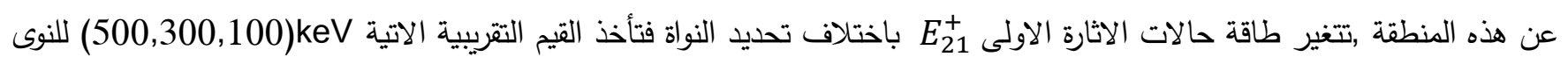

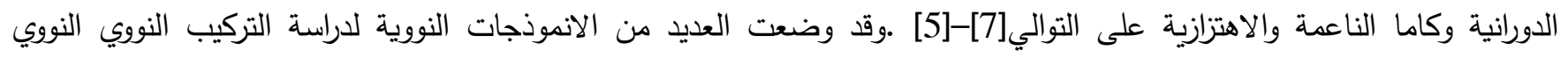

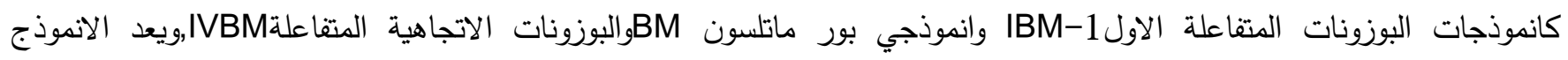

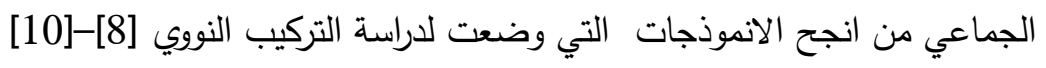

تم في هذا البحث التعرف على خصائص بعض نظائر الديسبروسيوم D DY D D الزوجية -الزوحية والتغير الذي يحصل فيها

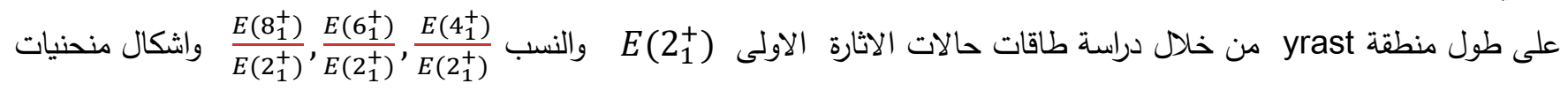


الانحناء الخلفي وطريقة E-GOS والعلاقة (I+2/I) كدالة للبرم وقيم الطاقات المحسوبة باستخدام المعادلات الخاصة لكل تحديد باستخدام انموذج البوزونات المتفاعلة الاول1BM-1 وانموذجي بور وماتلسون BM والبوزونات الاتجاهية المتفاعلة IVBM وقد تماء تالت

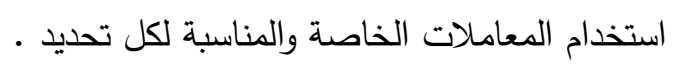

\section{2}

نجح انموذجاIVBM الذي اقترح من قبل [Ganev,2004] [11] في وصف حالات الطاقة الادنى للحزمة فروقات الطاقة بين الحزم المختلفة والذي يسمى بتأرجح الطاقة Staggering وفي دراسة التشوه الدوراني وكاما الناعمة اضافة الى النمط الاهتزازي [12] وقد نجح في دراسة الحزم ذات التماثل السالب والموجب بعد اختباره من قبل [13]. تعطى قيمة الطاقة للحزمة الارضية

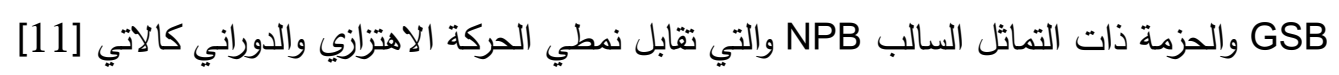

$\mathrm{E}(\mathrm{I})=\beta \mathrm{I}(\mathrm{I}+1)+\gamma \mathrm{I}$

$\mathrm{E}(\mathrm{I})=\beta \mathrm{I}(\mathrm{I}+1)+(\gamma+\eta) \mathrm{I}+\zeta$

حيث تحسب قيم الثوابت ر ر من خلال مواءمة Fitting للقيم التجريبية في الحزمة الارضية وتحسب قيم المعاملين ر, من مواءمة القيم التجريبية ايضا لكن للحزمة السالبة. اما بالنسبة لانموذج بور وماتلسون [14] فتحسب قيمة الطاقة للحزمة الارضية GSB والحزمة ذات التماثل السالب NPB من المعادلتين

$\mathrm{E}(\mathrm{I})=\mathrm{AI}(\mathrm{I}+1)+\mathrm{BI}^{2}(\mathrm{I}+1)^{2}+\mathrm{CI}^{3}(\mathrm{I}+1)^{3}$

$\mathrm{E}(\mathrm{I})=\mathrm{E}_{0}+\mathrm{AI}(\mathrm{I}+1)+\mathrm{BI}^{2}(\mathrm{I}+1)^{2}+\mathrm{CI}^{3}(\mathrm{I}+1)^{3}$

حيث ان A,B,C هي ثوابت يتم ايجادها من خلال مواءمة مع القيم العملية. والمعامل E تمثل طاقة راس الحزمة. في انموذج البوزونات المتفاعلة الاصدار الاول IBM-1 يعطى مؤثر دالة هاملتون بالعلاقة الاتية [15]

$\widehat{H}=\sum_{i=1}^{N} \varepsilon+\sum_{i<j}^{N} V_{i j}$

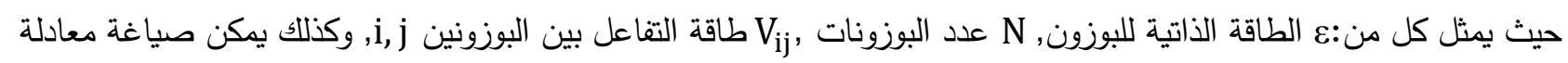
الهاملونين باستعمال صيغة متعدد القطبية [15]

(6) $\widehat{H}=\varepsilon \widehat{n}_{d}+a_{0} \hat{p} \cdot \hat{p}+a_{1} \widehat{L} \cdot \hat{L}+a_{2} \widehat{Q} \cdot \widehat{Q}+a_{3} \widehat{T}_{3} \cdot \widehat{T}_{3}+a_{4} \widehat{T}_{4} \cdot \widehat{T}_{4} \cdots$

حيث ان 
$\varepsilon=\varepsilon_{\mathrm{d}}+\varepsilon_{\mathrm{S}}$

اذ ان ع تمثل طاقة البوزونات الذاتية واذا ما اعتبر 0 = $=0$ بذلك تكون طاقة البوزونات الذاتية ع مساوية لطاقة بوزونات d أي انه

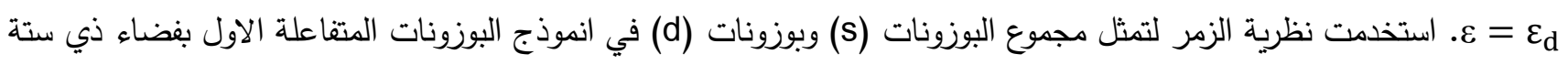

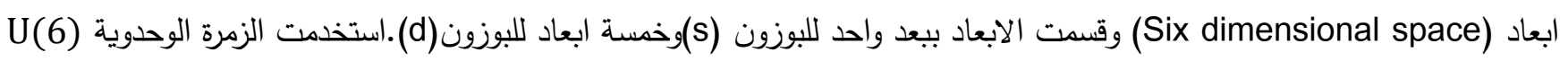

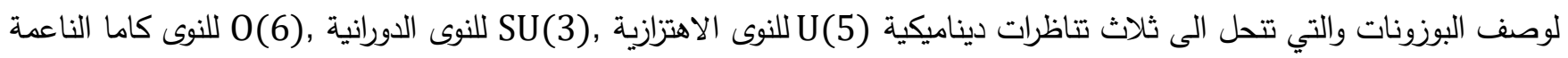

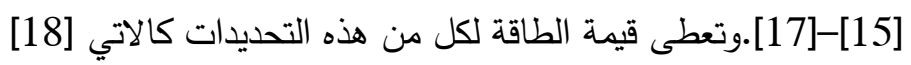

(8)U(5): $E\left(\varepsilon, n_{d}, \lambda, \mu, v, L\right)=\varepsilon n_{d}+K_{1} n_{d}\left(n_{d}+4\right)+K_{4} v(v+3)+K_{5} L(L+1)$

(9) $O(6): \quad E(N, \sigma, \tau, L)=K_{3}(N-\sigma)(N+\sigma+4)+K_{4} \tau(\tau+3)+K_{5} L(L+1)$

(10)SU(3): $E(\lambda, \mu, L)=K_{2}\left(\lambda^{2}+\mu^{2}+\lambda \mu+3 \lambda\right)+K_{5} L(L+1)$

في بعض النوى قد يحدث تغير درماتيكي في عزم القصور الذاتي مما يتسبب بانخفاض قيمة الطاقة الدورانية لانتقال طاقة كاما من الحالة (I) الى الحالة (I - 2 يتسبب هذا السلوك في حدوث ظاهرة الانحناء الخلفي الذي يظهر جلياً في النوى التي تعاني تغيراً في

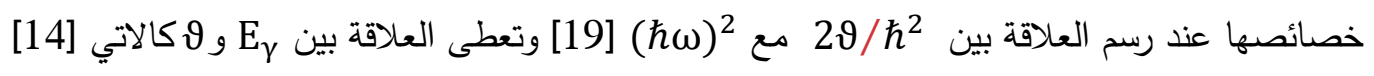
$(11) 2 \vartheta / \hbar^{2}=\frac{4 \mathrm{I}-2}{\mathrm{E}_{\gamma}}$

والعلاقة بين E و ع كالاتي [20]

$$
(12) \hbar \omega=\frac{E_{\gamma}}{\sqrt{I(I+1)}-\sqrt{(I-2)(I-1)}}
$$

ومن المثير للاهتمام مناقشة مستويات الطاقة من خلال رسم النسب(I) مهمة جدا في تحديد تركيبة النواة بالإضافة الى تحديد التغيرات كدالة للبرم لذا فان منحني E-GOS للنوى الاهتزازية تهبط بسرعة من

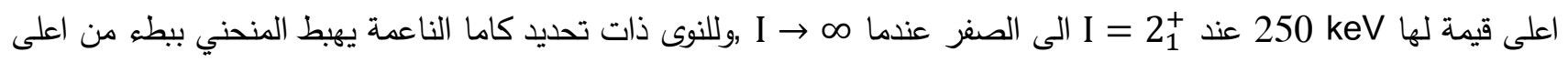
قيمة لها

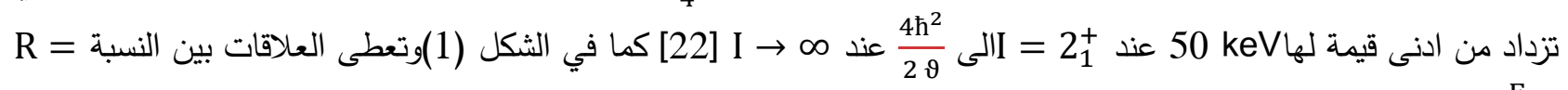

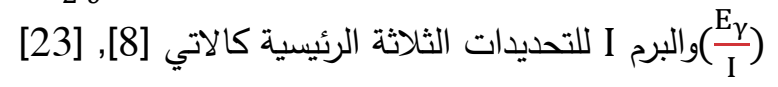

$\mathrm{U}(5): \mathrm{R}=\frac{\hbar \omega}{\mathrm{I}} \rightarrow 0 \quad$ When $\mathrm{I} \rightarrow \infty$

$\mathrm{O}(6): \mathrm{R}=\frac{\mathrm{E}\left(2_{1}^{+}\right)}{4}\left(1+\frac{2}{\mathrm{I}}\right) \rightarrow \frac{\mathrm{E} 2_{1}^{+}}{4}$ When $\mathrm{I} \rightarrow \infty$

$\mathrm{SU}(3): R=\frac{\hbar^{2}}{2 \vartheta}\left(4-\frac{2}{\mathrm{I}}\right) \rightarrow \frac{4 \hbar^{2}}{2 \vartheta} \quad$ When $\mathrm{I} \rightarrow \infty$ 


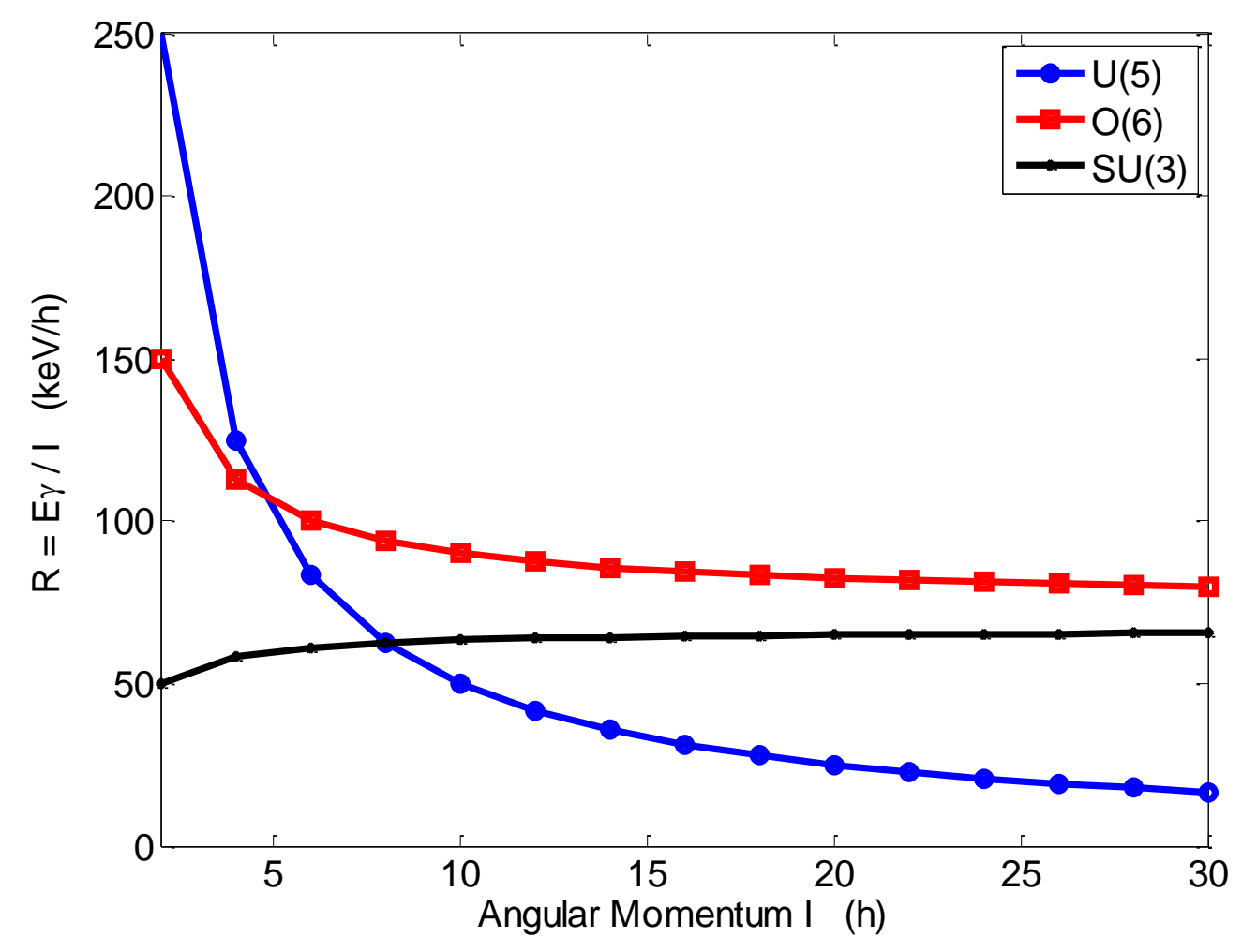

الثكل(1) : منحني E- GOS للحالات القياسية الثلاثة [22]

ولدينا النسبة بين قيم الطاقات لحالتين متتاليتين (I,I+2) والتي بدورها تزودنا بمعلومات كافية عن خصائص النواة وعند حالات مختلفة

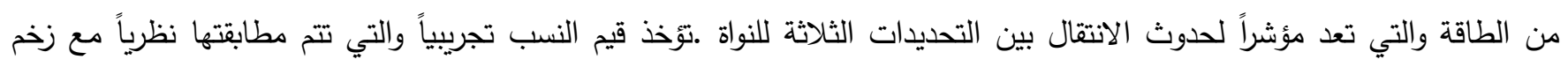

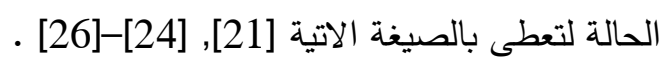

$r\left(\frac{I+2}{I}\right)=\left[R\left(\frac{I+2}{I}\right)_{\exp }-\left(\frac{I+2}{I}\right)\right] \times \frac{I(I+1)}{2(I+2)}$

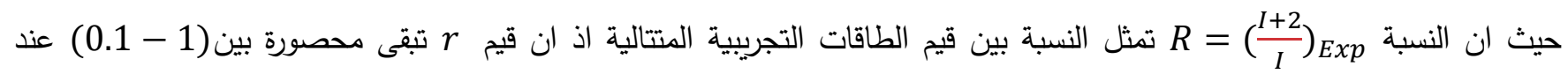

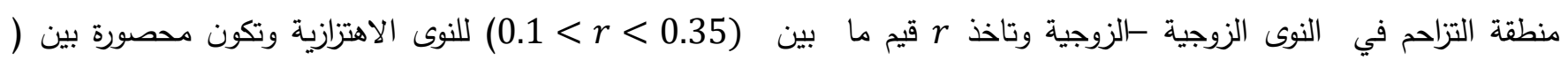

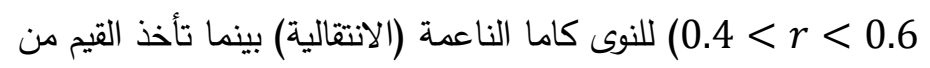

$$
\begin{aligned}
& \text { (26) } \\
& \text { 3. النتائج والمناقثة }
\end{aligned}
$$


تمتلك نواة الديسبروسيوم DY DY (66) بروتونا اما نظائرها 156 الزوجية - الزوجية تمتلك من (86) الى (90) نيترونا. في هذا البحث تم دراسة مواقع حالات الاثارة الاول(1) $\frac{E\left(8_{1}^{+}\right)}{E\left(2_{1}^{+}\right)}, \frac{E\left(6_{1}^{+}\right)}{E\left(2_{1}^{+}\right)}, \frac{E\left(4_{1}^{+}\right)}{E\left(2_{1}^{+}\right)}$ومقارنتها مع القيم الخاصة بالتحديدات الثلاثة الرئيسية كما في الجدول (1).

\begin{tabular}{|c|c|c|c|c|}
\hline & & الاراسة & & \\
\hline ISOTOP & $E 2_{1}^{+}$ & $R=E 4_{1}^{+} / E 2_{1}^{+}$ & $E 6_{1}^{+} / E 2_{1}^{+}$ & $E 8_{2}^{+} / E 2_{1}^{+}$ \\
\hline$\cup(5)$ & 500 & 2.0 & 3.0 & 4.0 \\
\hline $\mathrm{O}(6)$ & 300 & 2.5 & 4.5 & 7 \\
\hline $\mathrm{SU}(3)$ & 100 & 3.3 & 7 & 12 \\
\hline${ }^{152} \mathrm{DY}$ & 613.83 & 2.05 & 3.16 & 3.97 \\
\hline${ }^{154} \mathrm{DY}$ & 334.34 & 2.23 & 3.66 & 5.22 \\
\hline${ }^{156} \mathrm{DY}$ & 137.77 & 2.93 & 5.59 & 8.82 \\
\hline
\end{tabular}

وتم الحصول على معلومات اولية عن خصائص نظائر 156-156 الزوجية-الزوجية لكنها غير كافية, وللتأكد من تلك المعلومات تم

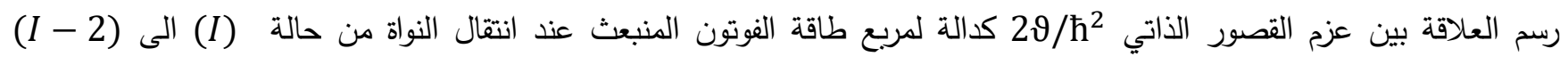
154-156DY بينما

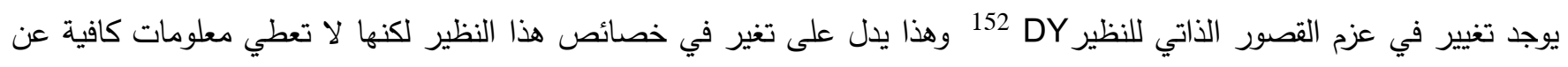

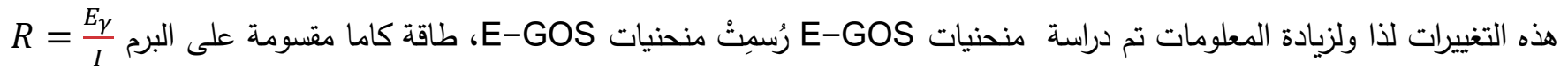
دالة للبرمI [22] للنظائر قيد الدراسة في الثكل(3) وتم مقارنتها مع الهنحنيات القياسية للتحديدات الثلاثة الرئيسية (الاهتزازية وكاما الناعمة والدورانية)، ويتضح من الثكل ان هنالك نقصان سريع من اقصى التحديد الاهتزازي للنظير 152 ويستمر بالهبوط السريع

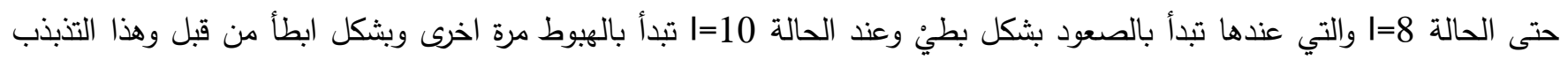
يدل على الخصائص الاهتزازية الغير نقية لهذا النظير اما بالنسبة للنظير هناللك هبوط سريع من اقصى تحديد كاما الناعمة

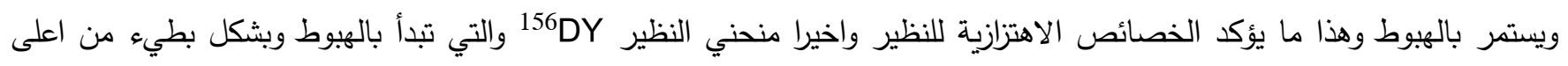
التحديد الدوراني ويستمر حتى الحالة 22=ا ومن ثم تستقر بعدها وهذا ما يؤكد تحديد خصائص كاما الناعمة للنظير لهريز 

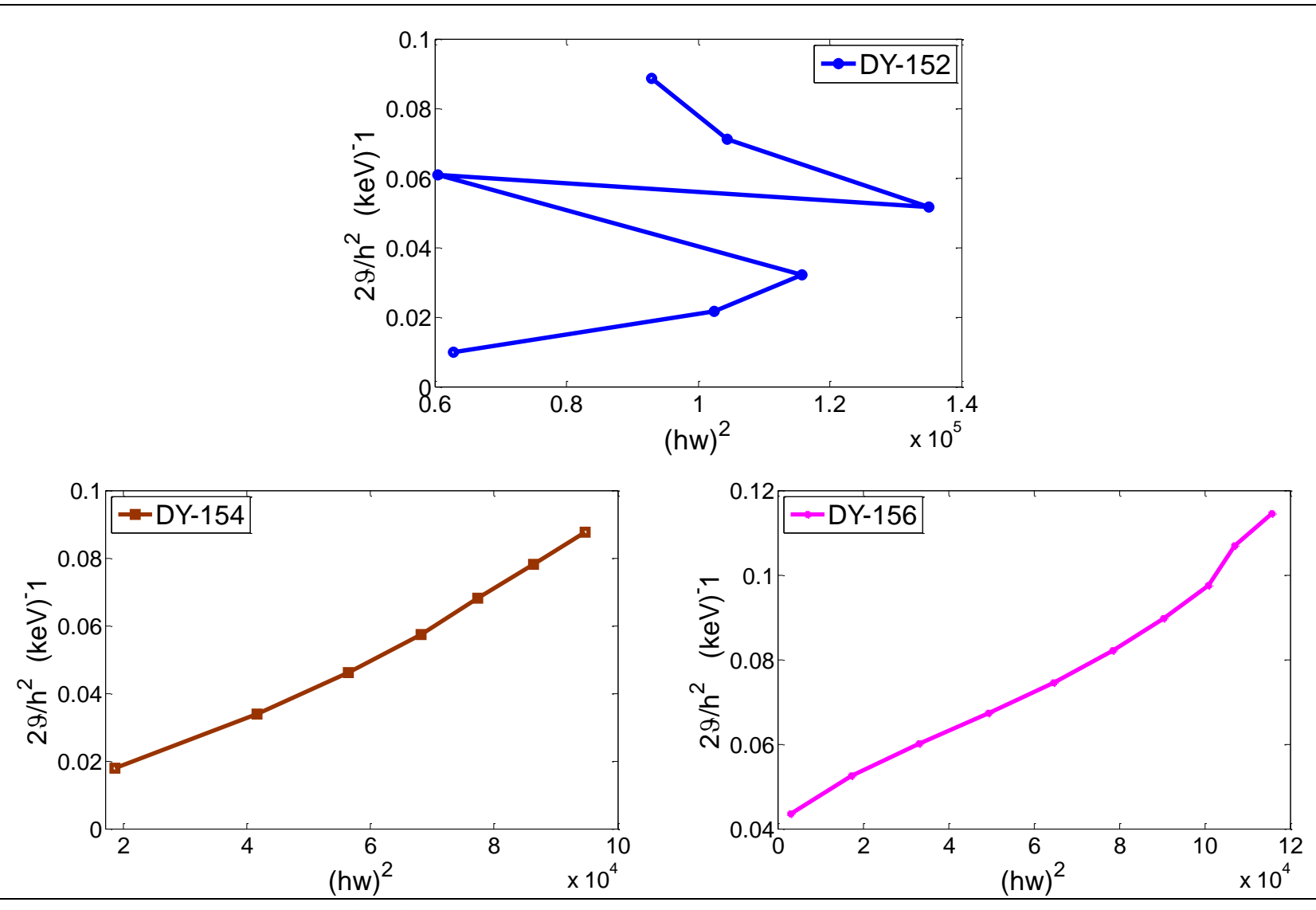

152-156DY الثكل (2):عزم القصور الذاتي دالة لمربع الطاقة الدورانية لنظائر x)

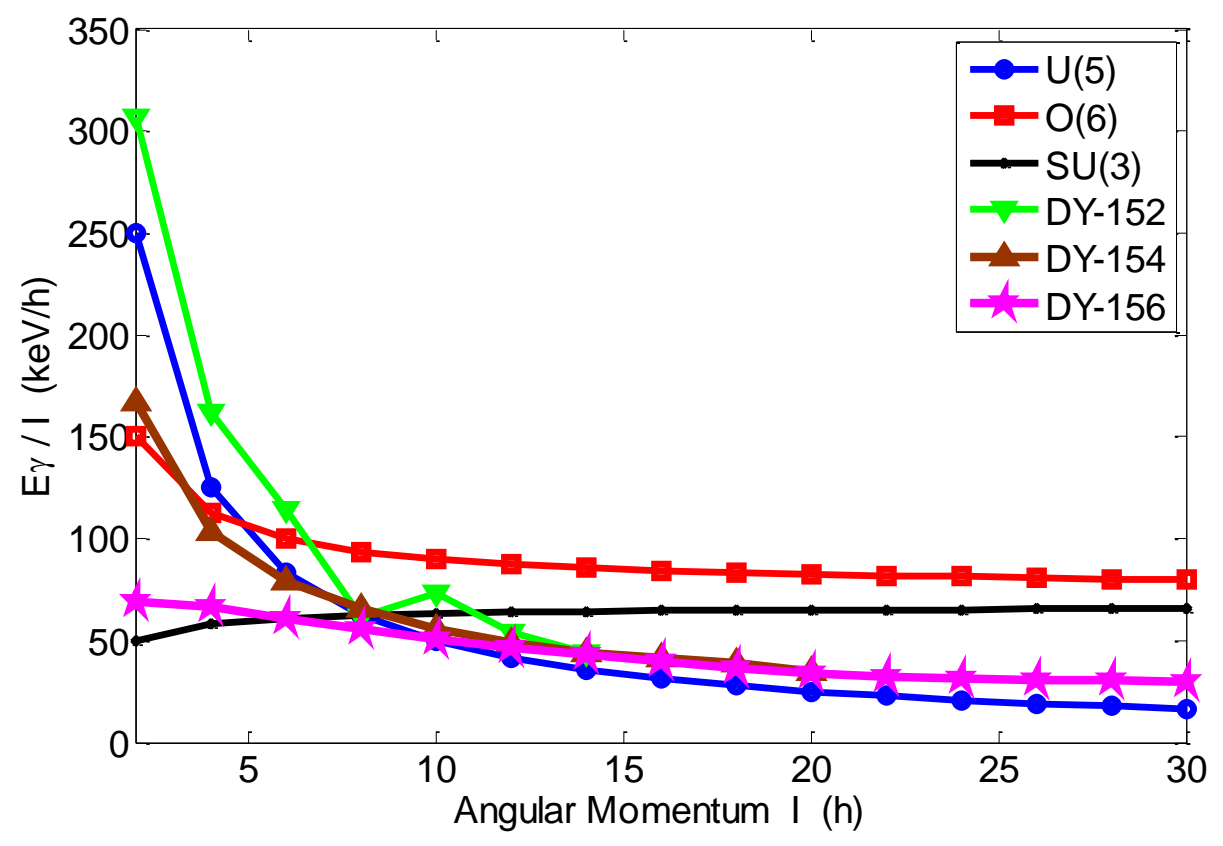

الثكل (3): منحنيات E-GOS لنظائر 152-156 DY مقارنة مع المنحنيات القياسية للتحديدات الثلاثة الرئيسية 


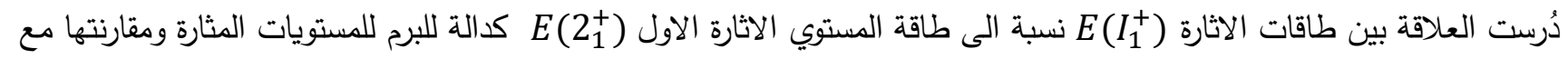

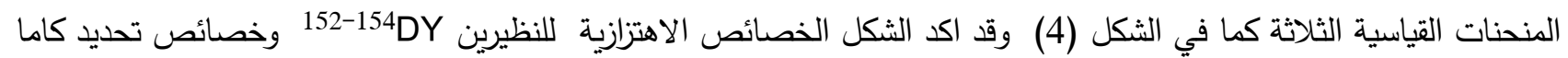
${ }^{156 D Y}$ الناعمة للنظير

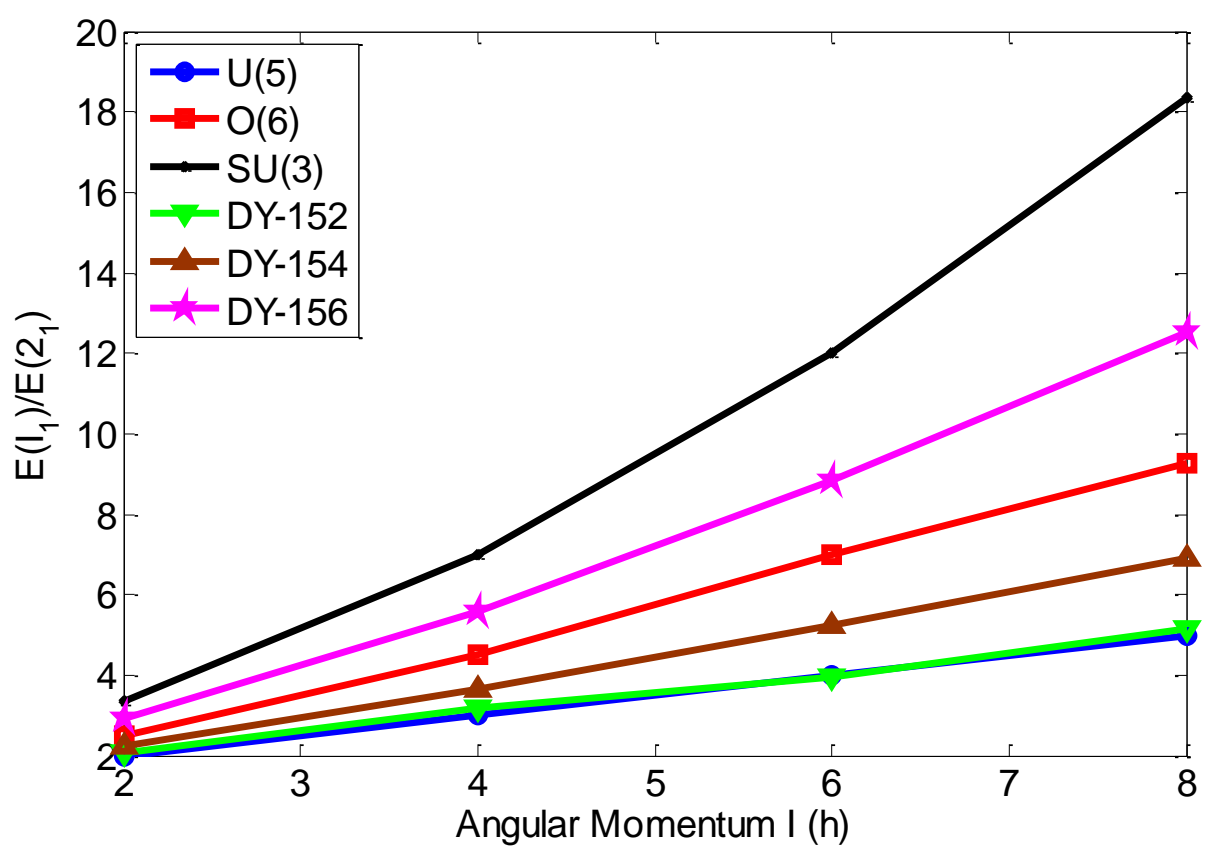

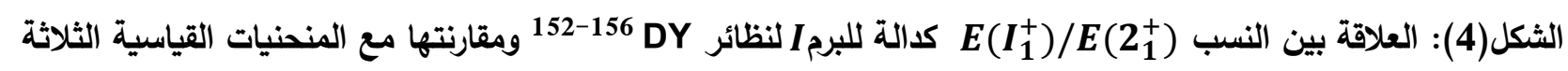

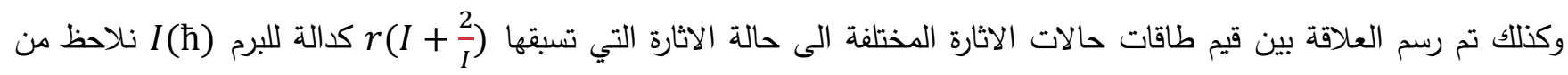

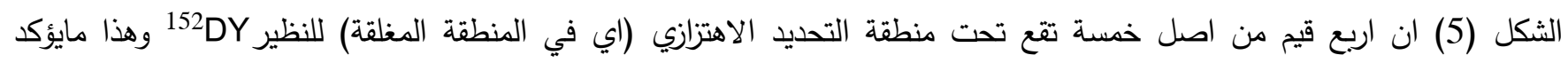

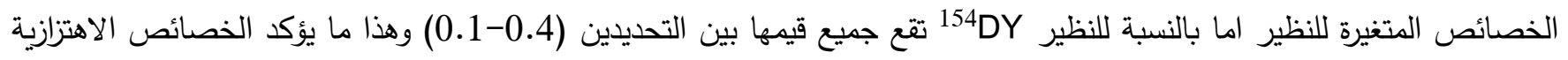

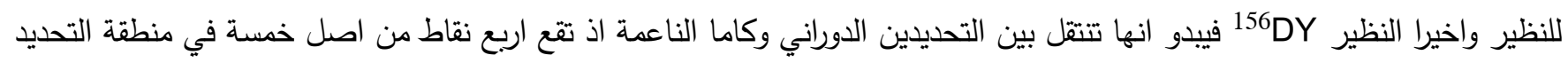

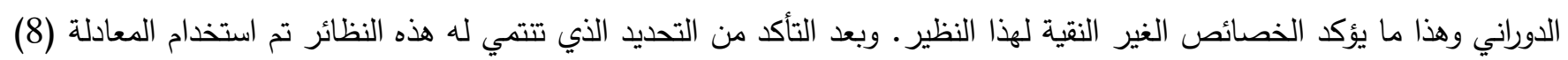

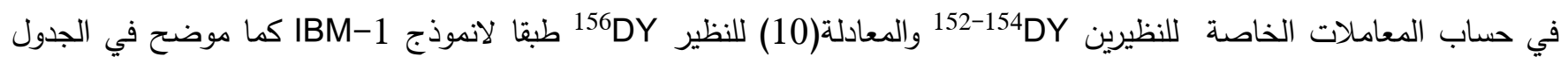

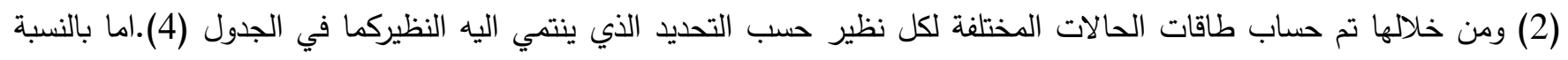

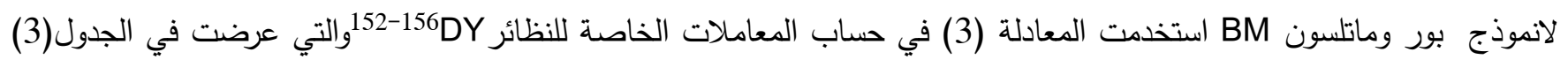

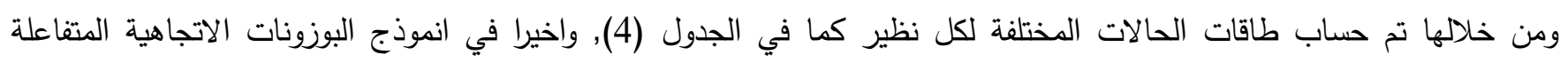

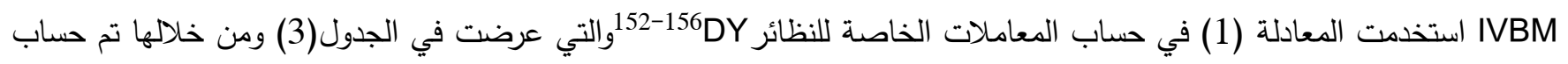

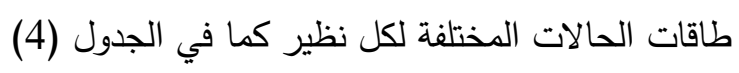

طبقا لانموذج البوزونات المتفاعلة الاول 1 - IBM تم حساب طاقات الحالات المختلفة من خلال استخدام المعادلة (11)القياسية

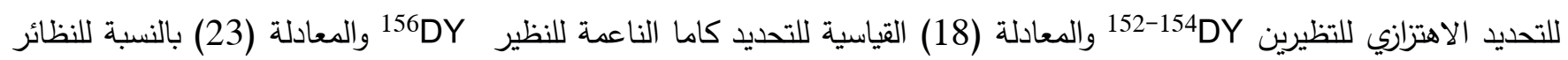


قيد الدراسة والمعادلة (22) بالنسبة لانموذج البوزونات الاتجاهية المتفاعلة IVBM ويتضح من خلال الجداول (4) نجد ان هنالك توافق جيد مع القيم العملية لطاقات الحالة الارضية.

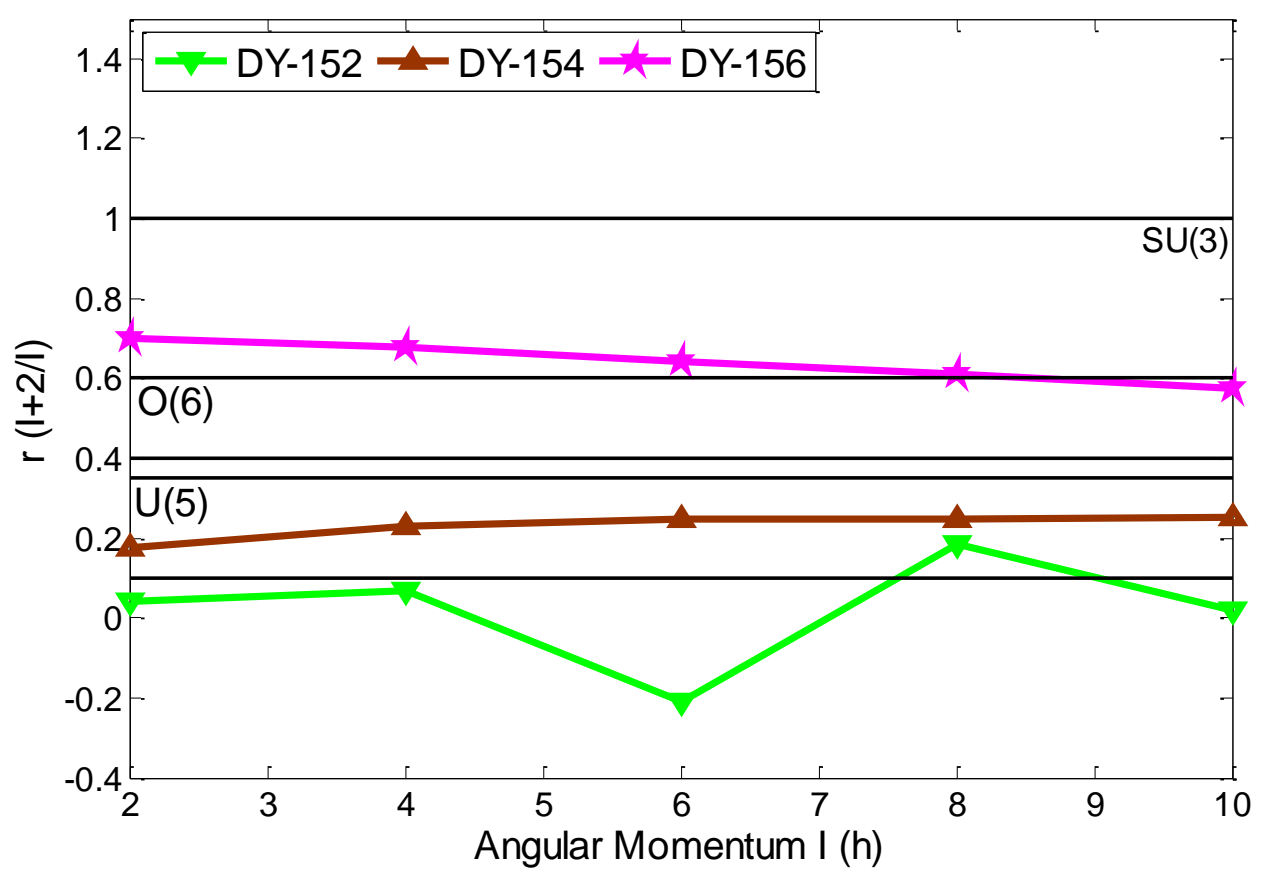

152-156DY كدالة للبرمI للنظكل(5) : النسبة بين

152-156DY للحزمة الارضية للنظائر IBM-1 الجدول (2):قيم المعاملات المستخدمة لحساب حالات الطاقة للانموذج

\begin{tabular}{cccccc}
\hline ISOTOPES & $N$ & $\varepsilon$ & $K_{1}$ & $K_{4}$ & $K_{5}$ \\
\hline${ }^{152} \mathrm{DY}$ & 10 & 561.35 & 12.36 & 7.71 & -4.67 \\
${ }^{154} \mathrm{DY}$ & 11 & 276.65 & 9.8 & 8.66 & 1.01 \\
& & & & & \\
${ }^{156} \mathrm{DY}$ & 12 & ----- & ----- & 55.14 & -4.71 \\
& & & & & \\
\hline
\end{tabular}


Journal of Education and Science (ISSN 1812-125X), Vol: 30, No: 4, 2021 (94-105)

الجدول (3):قيم المعاملات المستخدمة لحساب حالات الطاقة في الانموذجين MB و IVBM للحزمة الارضية للنظائر I56

\begin{tabular}{rcc|ccc}
\hline Isotopes & \multicolumn{2}{c|}{ BM } & & \multicolumn{2}{c}{ IVBM } \\
& A & B & C & $\beta$ & $\gamma$ \\
\hline & & & & & \\
${ }^{152} \mathrm{DY}$ & $5.8324 \mathrm{e}+001$ & $3.5834 \mathrm{e}-001$ & $8.6701 \mathrm{e}-004$ & 0.3438 & 311.9461 \\
${ }^{154} \mathrm{DY}$ & $3.3047 \mathrm{e}+001$ & $1.2971 \mathrm{e}-001$ & $2.3887 \mathrm{e}-004$ & 5.6249 & 166.3051 \\
${ }^{156} \mathrm{DY}$ & $1.9718 \mathrm{e}+001$ & $4.2382 \mathrm{e}-002$ & $6.467 \mathrm{e}-005$ & 9.0736 & $6.8926 \mathrm{e}+001$ \\
\hline
\end{tabular}

جدول (4): الطاقات العملية والمحسوبة بألانموذجات (BBM-1) , IVBM , بوحدات (MeV) للنظائر 152-156DY [27), .[28]

\begin{tabular}{|c|c|c|c|c|c|c|c|c|}
\hline \multicolumn{5}{|c|}{${ }^{152} \mathrm{DY}$} & \multicolumn{4}{|c|}{${ }^{154} \mathrm{DY}$} \\
\hline$I_{1}^{+}$ & $E_{\text {exp }}$ & $I V B M$ & $B M$ & $I B M-1$ & $E_{\text {exp }}$ & $I V B M$ & $B M$ & $I B M-1$ \\
\hline $2_{1}^{+}$ & 0.613 & 0.626 & 0.337 & 0.625 & 0.334 & 0.366 & 0.193 & 0.336 \\
\hline $4_{1}^{+}$ & 1.261 & 1.254 & 1.030 & 1.254 & 0.746 & 0.777 & 0.611 & 0.777 \\
\hline $6_{1}^{+}$ & 1.944 & 1.886 & 1.881 & 1.886 & 1.223 & 1.234 & 1.176 & 1.234 \\
\hline $8_{1}^{+}$ & 2.437 & 2.520 & 2.665 & 2.520 & 1.747 & 1.735 & 1.796 & 1.735 \\
\hline $10_{1}^{+}$ & 3.173 & 3.157 & 3.233 & 3.157 & 2.304 & 2.281 & 2.383 & 2.281 \\
\hline $12_{1}^{+}$ & 3.820 & 3.797 & 3.669 & 3.796 & 2.892 & 2.873 & 2.905 & 2.873 \\
\hline $14_{1}^{+}$ & 4.430 & 4.439 & 4.474 & 4.439 & 3.508 & 3.509 & 3.432 & 3.509 \\
\hline $16_{1}^{+}$ & ----- & 5.084 & 6.800 & ----- & 4.172 & 4.191 & 4.199 & 4.190 \\
\hline$I_{1}^{+}$ & \multicolumn{8}{|c|}{${ }^{156} \mathrm{DY}$} \\
\hline & \multicolumn{2}{|c|}{$E_{\text {exp }}$} & $I V B M$ & \multicolumn{2}{|c|}{$B M$} & \multicolumn{3}{|c|}{$I B M-1$} \\
\hline
\end{tabular}

$\begin{array}{ccccc}2_{1}^{+} & 0.137 & 0.192 & 0.116 & 0.192 \\ 4_{1}^{+} & 0.404 & 0.457 & 0.377 & 0.457 \\ 6_{1}^{+} & 0.770 & 0.794 & 0.758 & 0.794 \\ 8_{1}^{+} & 1.215 & 1.204 & 1.224 & 1.204 \\ 10_{1}^{+} & 1.725 & 1.687 & 1.742 & 1.687 \\ 12_{1}^{+} & 2.285 & 2.242 & 2.290 & 2.242 \\ 14_{1}^{+} & 2.887 & 2.870 & 2.870 & 2.870 \\ 16_{1}^{+} & 3.523 & 3.570 & 3.529 & 3.570\end{array}$


من منحنيات الأنحناء الخلفي للنظائر قيد الدراسة يتضح ان هذه النظائر لاتحدث فيها هذه الظاهرة سوى للنظير 152 15هذا يؤكد انه

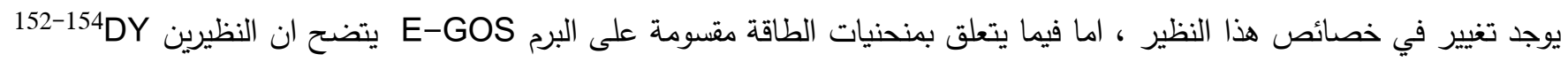

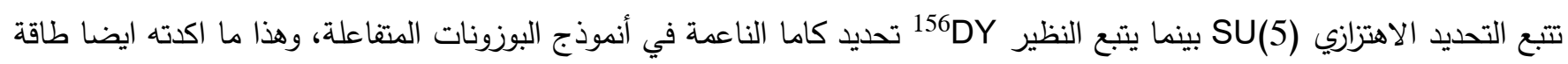

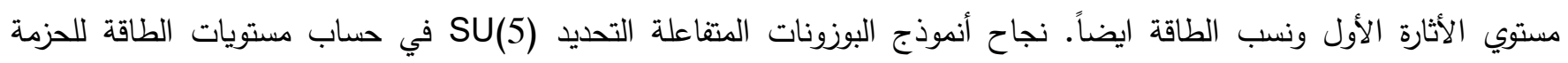

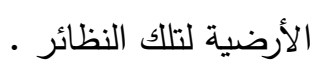

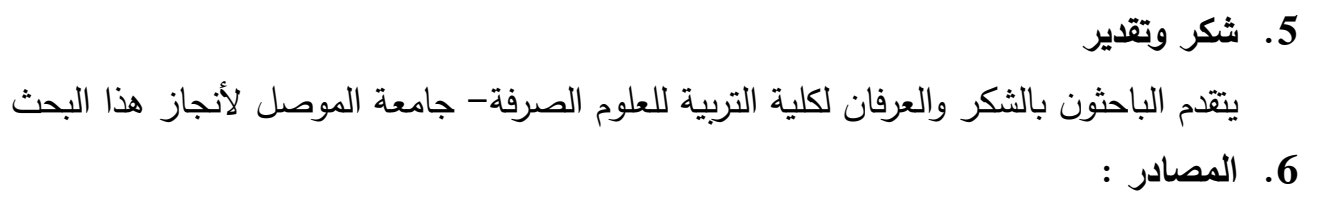

[1] B. L. Cohen, Concepts of nuclear physics. McGraw Hill Education, 1971.

[2] W. Greiner and J. A. Maruhn, Nuclear Models. Springer Berlin Heidelberg, 1996.

[3] J. E. Grosse, F. S. Stephens, and R. M. Diamond, "Test of Backbending Models Using Odd-A Nuclei," phys. Rev. Lett., vol. 31, no. 9, pp. 840-843, 1973.

[4] P. von Brentano, A. Gelberg, and S. Harissopulos, "Test of the $\mathrm{O}(6)$ character ofnuclei near A $=130$," phys. Rev. Lett., vol. 38, no. 5, pp. 6-8, 1988 .

[5] A. Bohr and B. R. Mottelson, "Nuclear Structure Vol. II," WA Benjamin Inc., New York, Amsterdam, 1975.

[6] Y. Y. Kassim, M. A. Al-Jubbori, I. M. Ahmed, H. Y. Abdullah, and F. I. Sharrad, "Microscopic description of 170Er, 172 Yb, 174Hf, 176W isotones," IOP Conf. Ser. Mater. Sci. Eng., vol. 928, no. 7, 2020.

[7] H. H. Kassim, A. A. Abd-Aljbar, M. A. Al-Jubbori, H. Y. Abdullah, I. Hossain, and F. I. Sharrad, "Properties of O(6)-U(5) transition symmetry for 122-124Cd isotopes in IBM," IOP Conf. Ser. Mater. Sci. Eng., vol. 928, no. 7, 2020.

[8] A. Bohr and B. R. Mottelson., "Physics of $\mathrm{N}=\mathrm{Z}$ and $\mathrm{N}=\mathrm{Z}+1$ nuclei in the $\mathrm{A}=80-100$ region," Mat. Fys. Med, vol. 27, 1953.

[9] M. A. Al-Jubbori et al., "Critical Point of the 152Sm, 154Gd, and 156Dy Isotones," Phys. At. Nucl., vol. 82, no. 3, pp. 201-211, 2019.

[10] H. H. Kassim, M. A. Al-Jubbori, M. M. Yousif, I. M. Ahmed, and F. I. Sharrad, "Nuclear Structure and Energy Levels of 158Er, $160 \mathrm{Yb}$ and 162Hf Isotones," IOP Conf. Ser. Mater. Sci. Eng., vol. 928, no. 7, 2020.

[11] H. Ganev, V. P. Garistov, and A. I. Georgieva, "Description of the ground and octupole bands in the symplectic extension of the interacting vector boson model," Phys. Rev. C, vol. 69, no. 1, p. 7, 2004. 
[12] H. G. Ganev, "Triaxiality in the IVBM," Nucl. Theory, vol. 32, pp. 204-214, 2013.

[13] V. Garistov, A. Georgieva, and T. Shneidman, "Description of Alternating Parity Bands in Deformed Even-Even Nuclei in the Symplectic Extension of the Interacting Vector Boson Model," Bulg. J. Phys, vol. 40, pp. 1-16, 2013.

[14] Samuel S M Wong, Introductory Nuclear Physics. London: Prentice-Hall intern. ed. London, 1990.

[15] A. Arima and F. Iachello, "Interacting boson model of collective nuclear states IV. The $\mathrm{O}(6)$ limit," Ann. Phys. vol. 123, no. 2, pp. 468-492, Dec. 1979.

[16] A. Arima and F. Iachello, "Interacting boson model of collective states I. The vibrational limit," Ann. Phys. vol. 281, no. 1-2, pp. 2-64, Apr. 2000.

[17] A. Arima and F. Iachello, "Interacting boson model of collective nuclear states II. The rotational limit," Ann. Phys. vol. 111, no. 1, pp. 201-238, 1978.

[18] F. Iachello and A. Arima, The Interacting Boson Model. Cambridge University Press, 1987.

[19] A. Johnson, H. Ryde, and J. Sztarkier, "Evidence for a 'singularity' in the nuclear rotational band structure,” Phys. Lett. B, vol. 34, no. 7, pp. 605-608, Apr. 1971.

[20] R. A. Sorensen, "Nuclear Moment of Inertia at High Spin," Rev. Mod. Phys., vol. 45, no. 3, pp. 353-377, 1973.

[21] I. Mamdouh and M. Al-Jubbori, "The rotational-vibrational properties of 178-188Os isotopes," Indian J. Phys., vol. 89, no. 10, pp. 1085-1091, 2015.

[22] P. H. Regan et al., "Signature for Vibrational to Rotational Evolution Along the Yrast Line," Phys. Rev. Lett., vol. 90, no. 15, p. 4, 2003.

[23] Scharff-Goldhaber and J. Gertrude and Weneser, "System of Even-Even Nuclei," Phys. Rev., vol. 98, no. 1, pp. 212-214, 1955.

[24] M. A. Al-Jubbori, H. H. Kassim, F. I. Sharrad, and I. Hossain, "Nuclear structure of even 120136Ba under the framework of IBM, IVBM and new method (SEF)," Nucl. Phys. A, vol. 955, pp. 101-115, Nov. 2016.

[25] D. Bonatsos, L. D. Skouras, and J. Rikovska, "Successive energy ratios in medium- and heavymass nuclei as indicators of different kinds of collectivity," vol. 43, no. 3, pp. 952-956, 1991.

[26] A. M. Khalaf and A. M. Ismail, "Structure shape evolution in lanthanide and actinide nuclei," PROGRESS, p. 98, 2013.

[27] M. J. Martin, "Nuclear Data Sheets for A = 152," Nucl. Data Sheets, vol. 114, no. 11, pp. 1497 1847, Nov. 2013.

[28] C. W. Reich, "Nuclear Data Sheets for A = 156," Nucl. Data Sheets, vol. 113, no. 11, pp. 2537 2840, Nov. 2012. 